IJMMS 31:12 (2002) 751-760

PII. S0161171202109276

http://ijmms.hindawi.com

(c) Hindawi Publishing Corp.

\title{
ON THE RELATION BETWEEN INTERIOR CRITICAL POINTS OF POSITIVE SOLUTIONS AND PARAMETERS FOR A CLASS OF NONLINEAR BOUNDARY VALUE PROBLEMS
}

\section{G. A. AFROUZI and M. KHALEGHY MOGHADDAM}

Received 10 September 2001

\begin{abstract}
We consider the boundary value problem $-u^{\prime \prime}(x)=\lambda f(u(x)), x \in(0,1) ; u^{\prime}(0)=0$; $u^{\prime}(1)+\alpha u(1)=0$, where $\alpha>0, \lambda>0$ are parameters and $f \in c^{2}[0, \infty)$ such that $f(0)<0$. In this paper, we study for the two cases $\rho=0$ and $\rho=\theta$ ( $\rho$ is the value of the solution at $x=0$ and $\theta$ is such that $F(\theta)=0$ where $F(s)=\int_{0}^{s} f(t) d t$ the relation between $\lambda$ and the number of interior critical points of the nonnegative solutions of the above system.
\end{abstract}

2000 Mathematics Subject Classification: 34B15.

1. Introduction. We consider the two point boundary value problem with NeumannRobin boundary conditions

$$
\begin{gathered}
-u^{\prime \prime}(x)=\lambda f(u(x)), \quad x \in(0,1), \\
u^{\prime}(0)=0, \\
u^{\prime}(1)+\alpha u(1)=0,
\end{gathered}
$$

where $\alpha>0, \lambda>0$ are parameters, $f \in c^{2}[0, \infty)$ and $f(0)<0$, and we will assume that there exist $\beta, \theta>0$ such that $f(s)<0$ on $[0, \beta), f(\beta)=0, f^{\prime}(s) \geq 0, f^{\prime \prime}(s)>0$, $\lim _{s \rightarrow \infty}(f(s) / s)=\infty$, and $F(\theta)=0$ where $F(s)=\int_{0}^{s} f(t) d t$. It is proved in [1, Theorems 3.4.1(a) and 3.4.1(b)] that for any $n=0,1,2, \ldots, \alpha \in(0, \infty), \rho=\theta(\rho=0),(1.1),(1.2)$, and (1.3) have exactly two nonnegative solutions $u_{2 n, i}\left(u_{2 n+1, i}\right), i=1,2$ with $2 n$ (and $2 n+1)$ interior critical points. Also it is shown in [3, Theorem 1.4] that for the following Dirichlet boundary conditions

$$
\begin{gathered}
-u^{\prime \prime}(x)=\lambda f(u(x)), \quad x \in(0,1), \\
u(0)=0=u(1),
\end{gathered}
$$

where $n$ is a positive integer, there exists $\lambda^{*}>0$ such that (1.4) has a unique nonnegative solution with $n$ interior zeros if and only if $\lambda=(n+1)^{2} \lambda^{*}$. Equation (1.1) in the cases Neumann and Dirichlet-Robin boundary conditions have been studied in [2, 4], respectively. We discuss the relation between interior critical points of nonnegative solutions and $\lambda$ 's for problem (1.1), (1.2), and (1.3) for the case $\rho=\theta$ in Section 2, and for the case $\rho=0$ in Section 3. Finally, in Section 4 we compare $\lambda$ 's in two cases $\rho=\theta$ and $\rho=0$ for any $n=0,1,2, \ldots$. 


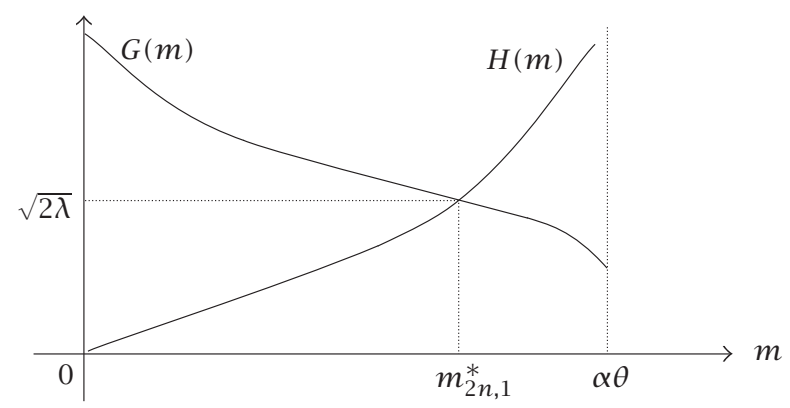

FIGURE 2.1

2. The case $\rho=\theta$. In [1] it has been established that for $\alpha \in(0, \infty), \rho=\theta$, and $n=0,1,2, \ldots$, there exists a unique number $m_{2 n, 1}^{*} \in(0, \alpha \theta)$ such that

$$
G\left(m_{2 n, 1}^{*}\right)=H\left(m_{2 n, 1}^{*}\right),
$$

where

$$
\begin{aligned}
& G(m)=\int_{m / \alpha}^{\theta} \frac{1}{\sqrt{-F(s)}} d s+2 n \int_{0}^{\theta} \frac{1}{\sqrt{-F(s)}} d s, \quad m \in(0, \alpha \theta), \\
& H(m)=\frac{m}{\sqrt{-F(m / \alpha)}}, \quad m \in(0, \alpha \theta) .
\end{aligned}
$$

So we obtain $\lambda=\lambda_{2 n, 1}\left(\theta, m_{2 n, 1}^{*}\right)$ such that $\sqrt{2 \lambda}=G\left(m_{2 n, 1}^{*}\right)=H\left(m_{2 n, 1}^{*}\right)$ (see Figure 2.1), that is,

$$
\sqrt{\lambda}=\frac{1}{\sqrt{2}} \int_{m / \alpha}^{\theta} \frac{1}{\sqrt{-F(s)}} d s+\frac{2 n}{\sqrt{2}} \int_{0}^{\theta} \frac{1}{\sqrt{-F(s)}} d s \quad m=m_{2 n, 1}^{*} .
$$

Thus (1.1), (1.2), and (1.3) have exactly a nonzero solution $u_{2 n, 1}$ with $2 n$ interior critical points where $u_{2 n, 1}^{\prime}(1)=-m_{2 n, 1}^{*}$ and $u_{2 n, 1}^{\prime}(0)=\theta$ at $\lambda=\lambda_{2 n, 1}\left(\theta, m_{2 n, 1}^{*}\right)$. Also, the equation

$$
\sqrt{\lambda}=\frac{2 n+1}{\sqrt{2}} \int_{0}^{\theta} \frac{1}{\sqrt{-F(s)}} d s
$$

has a unique solution $\lambda=\lambda_{2 n, 2}(\theta, 0)$ such that for this $\lambda$ problem, (1.1), (1.2), and (1.3) have exactly a nonnegative solution $u_{2 n, 2}$ with $2 n$ interior critical points such that $u_{2 n, 2}^{\prime}(1)=0$ and $u_{2 n, 2}(0)=\theta$.

In [1], it is proved that

$$
\lambda_{2 n, 1}\left(\theta, m_{2 n, 1}^{*}\right)<\lambda_{2 n, 2}(\theta, 0)<\lambda_{2(n+1), 1}\left(\theta, m_{2(n+1), 1}^{*}\right) .
$$

Now we are ready to prove the main theorem of this section. 


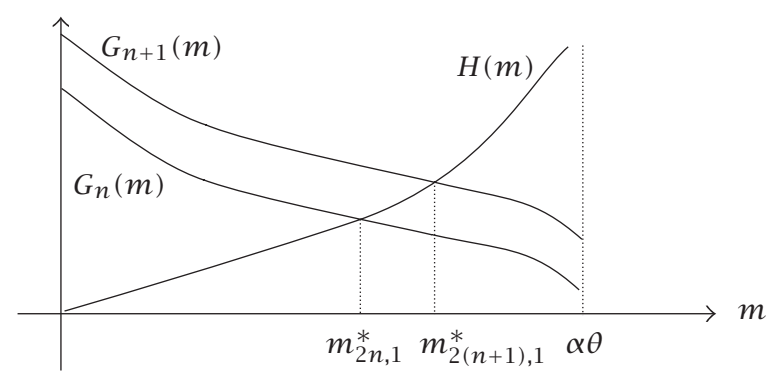

FIGURE 2.2

THEOREM 2.1. Let $n=0,1,2, \ldots$, then

$$
\lambda_{2 n, 2}-\lambda_{2 n, 1}<\lambda_{2(n+1), 2}-\lambda_{2(n+1), 1},
$$

that is, $n \mapsto \lambda_{2 n, 2}-\lambda_{2 n, 1}$ is a strictly increasing function.

Proof. Since $G(m)$ is dependent on $n$, so we write it by $G_{n}(m)$, that is,

$$
G_{n}(m)=\int_{m / \alpha}^{\theta} \frac{1}{\sqrt{-F(s)}} d s+2 n \int_{0}^{\theta} \frac{1}{\sqrt{-F(s)}} d s, \quad m \in(0, \alpha \theta)
$$

So it is easy to see that $\left\{G_{n}(m)\right\}_{n=0}^{\infty}$ is a strictly increasing sequence of $n$ for every $m \in(0, \alpha \theta)$, that is,

$$
G_{n}(m)<G_{n+1}(m), \quad m \in(0, \alpha \theta)
$$

and we can easily see that

$$
m_{2 n, 1}^{*}<m_{2(n+1), 1}^{*}, \quad n=0,1,2, \ldots
$$

(see Figure 2.2). On the other hand, from (2.3) and (2.4) we have

$$
\begin{gathered}
\sqrt{\lambda_{2 n, 2}}-\sqrt{\lambda_{2 n, 1}}=\frac{1}{\sqrt{2}} \int_{0}^{\theta} \frac{1}{\sqrt{-F(s)}} d s-\frac{1}{\sqrt{2}} \int_{m / \alpha}^{\theta} \frac{1}{\sqrt{-F(s)}} d s, \quad m=m_{2 n, 1}^{*}, \\
\sqrt{\lambda_{2(n+1), 2}}-\sqrt{\lambda_{2(n+1), 1}}=\frac{1}{\sqrt{2}} \int_{0}^{\theta} \frac{1}{\sqrt{-F(s)}} d s-\frac{1}{\sqrt{2}} \int_{m / \alpha}^{\theta} \frac{1}{\sqrt{-F(s)}} d s, \quad m=m_{2(n+1), 1}^{*} .
\end{gathered}
$$

Thus combining (2.9) and (2.10) we obtain

$$
\sqrt{\lambda_{2 n, 2}}-\sqrt{\lambda_{2 n, 1}}<\sqrt{\lambda_{2(n+1), 2}}-\sqrt{\lambda_{2(n+1), 1}} .
$$


Also combining (2.4) and (2.9) we have

$$
\begin{aligned}
& \sqrt{\lambda_{2 n, 2}}+\sqrt{\lambda_{2 n, 1}} \\
& =\frac{4 n+1}{\sqrt{2}} \int_{0}^{\theta} \frac{1}{\sqrt{-F(s)}} d s+\frac{1}{\sqrt{2}} \int_{m / \alpha}^{\theta} \frac{1}{\sqrt{-F(s)}} d s, \quad m=m_{2 n, 1}^{*}, \\
& \sqrt{\lambda_{2(n+1), 2}}+\sqrt{\lambda_{2(n+1), 1}} \\
& =\frac{4 n+5}{\sqrt{2}} \int_{0}^{\theta} \frac{1}{\sqrt{-F(s)}} d s+\frac{1}{\sqrt{2}} \int_{m / \alpha}^{\theta} \frac{1}{\sqrt{-F(s)}} d s, \quad m=m_{2(n+1), 1}^{*} .
\end{aligned}
$$

Since $0<m / \alpha<\theta$ for $m=m_{2 n, 1}^{*}$, so we have

$$
\int_{m / \alpha}^{\theta} \frac{1}{\sqrt{-F(s)}} d s<\int_{0}^{\theta} \frac{1}{\sqrt{-F(s)}} d s, \quad m=m_{2 n, 1}^{*},
$$

and then

$$
\frac{1}{\sqrt{2}} \int_{m / \alpha}^{\theta} \frac{1}{\sqrt{-F(s)}} d s+\frac{4 n+1}{\sqrt{2}} \int_{0}^{\theta} \frac{1}{\sqrt{-F(s)}} d s<\frac{1}{\sqrt{2}} \int_{0}^{\theta} \frac{1}{\sqrt{-F(s)}} d s+\frac{4 n+1}{\sqrt{2}} \int_{0}^{\theta} \frac{1}{\sqrt{-F(s)}} d s .
$$

Now from (2.12) and (2.15) we obtain

$$
\sqrt{\lambda_{2 n, 2}}+\sqrt{\lambda_{2 n, 1}}<\frac{4 n+2}{\sqrt{2}} \int_{0}^{\theta} \frac{1}{\sqrt{-F(s)}} d s .
$$

On the other hand, by the positivity of

$$
\frac{3}{\sqrt{2}} \int_{0}^{\theta} \frac{1}{\sqrt{-F(s)}} d s+\frac{1}{\sqrt{2}} \int_{m / \alpha}^{\theta} \frac{1}{\sqrt{-F(s)}} d s, \quad m=m_{2(n+1), 1}^{*},
$$

and also from (2.13) and (2.16) we obtain

$$
\sqrt{\lambda_{2 n, 2}}+\sqrt{\lambda_{2 n, 1}}<\sqrt{\lambda_{2(n+1), 2}}+\sqrt{\lambda_{2(n+1), 1}} .
$$

Now combining (2.11) and (2.18) we obtain

$$
\begin{aligned}
\left(\sqrt{\lambda_{2 n, 2}}-\sqrt{\lambda_{2 n, 1}}\right)\left(\sqrt{\lambda_{2 n, 2}}+\sqrt{\lambda_{2 n, 1}}\right) & <\left(\sqrt{\lambda_{2 n, 2}}+\sqrt{\lambda_{2 n, 1}}\right)\left(\sqrt{\lambda_{2(n+1), 2}}-\sqrt{\lambda_{2(n+1), 1}}\right) \\
& <\left(\sqrt{\lambda_{2(n+1), 2}}-\sqrt{\lambda_{2(n+1), 1}}\right)\left(\sqrt{\lambda_{2(n+1), 2}}+\sqrt{\lambda_{2(n+1), 1}}\right)
\end{aligned}
$$

and so,

$$
\lambda_{2 n, 2}-\lambda_{2 n, 1}<\lambda_{2(n+1), 2}-\lambda_{2(n+1), 1},
$$

thus, the proof is complete. 


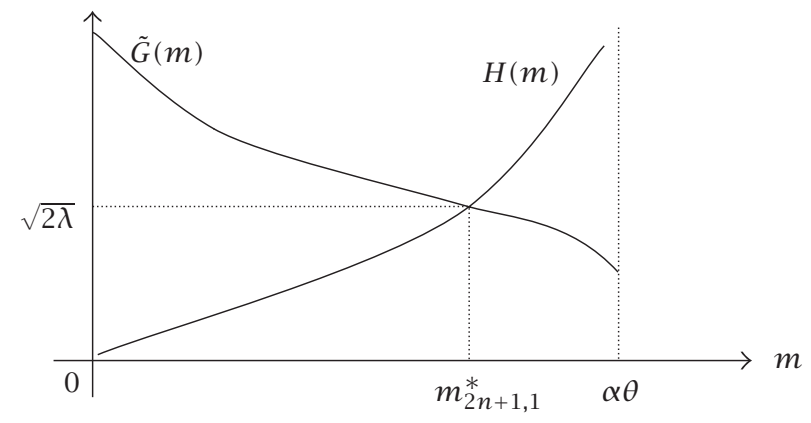

FIGURE 3.1

3. The case $\rho=0$. Also in [1] it has been established that for $\alpha \in(0, \infty), \rho=0$, and $n=0,1,2, \ldots$, there exists a unique number $m_{2 n+1,1}^{*} \in(0, \alpha \theta)$ such that

$$
\tilde{G}\left(m_{2 n+1,1}^{*}\right)=H\left(m_{2 n+1,1}^{*}\right),
$$

where

$$
\begin{aligned}
& \tilde{G}(m)=\int_{m / \alpha}^{\theta} \frac{1}{\sqrt{-F(s)}} d s+(2 n+1) \int_{0}^{\theta} \frac{1}{\sqrt{-F(s)}} d s, \quad m \in(0, \alpha \theta), \\
& H(m)=\frac{m}{\sqrt{-F(m / \alpha)}}, \quad m \in(0, \alpha \theta) .
\end{aligned}
$$

So we obtain $\lambda=\lambda_{2 n+1,1}\left(0, m_{2 n+1,1}^{*}\right)$ such that $\sqrt{2 \lambda}=\tilde{G}\left(m_{2 n+1,1}^{*}\right)=H\left(m_{2 n+1,1}^{*}\right)$ (see Figure 3.1), that is,

$$
\sqrt{\lambda}=\frac{1}{\sqrt{2}} \int_{m / \alpha}^{\theta} \frac{1}{\sqrt{-F(s)}} d s+\frac{2 n+1}{\sqrt{2}} \int_{0}^{\theta} \frac{1}{\sqrt{-F(s)}} d s, \quad m=m_{2 n+1,1}^{*} .
$$

Thus (1.1), (1.2), and (1.3) have exactly a nonzero solution $u_{2 n+1,1}$ with $2 n+1$ interior critical points where $u_{2 n+1,1}^{\prime}(1)=-m_{2 n+1,1}^{*}$ and $u_{2 n+1,1}^{\prime}(0)=0$ at $\lambda=\lambda_{2 n+1,1}\left(0, m_{2 n+1,1}^{*}\right)$. Also, the equation

$$
\sqrt{\lambda}=\frac{2(n+1)}{\sqrt{2}} \int_{0}^{\theta} \frac{1}{\sqrt{-F(s)}} d s
$$

has a unique solution $\lambda=\lambda_{2 n+1,2}(0,0)$ such that for this $\lambda$ problem, (1.1), (1.2), and (1.3) have exactly a nonnegative solution $u_{2 n+1,2}$ with $2 n+1$ interior critical points such that $u_{2 n+1,2}^{\prime}(1)=0$ and $u_{2 n+1,2}(0)=0$.

In [1], it is proved that

$$
\lambda_{2 n+1,1}\left(0, m_{2 n+1,1}^{*}\right)<\lambda_{2 n+1,2}(0,0)<\lambda_{2(n+1)+1,1}\left(0, m_{2(n+1)+1,1}^{*}\right) .
$$

Now we are ready to prove the main theorem of this section. 


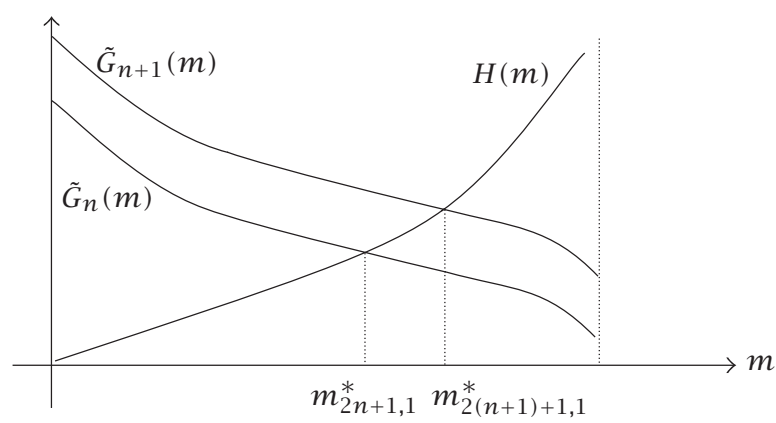

FIGURE 3.2

THEOREM 3.1. Let $n=0,1,2, \ldots$, then

$$
\lambda_{2 n+1,2}-\lambda_{2 n+1,1}<\lambda_{2(n+1)+1,2}-\lambda_{2(n+1)+1,1},
$$

that is, $n \mapsto \lambda_{2 n+1,2}-\lambda_{2 n+1,1}$ is a strictly increasing function.

Proof. Since $\tilde{G}(m)$ is dependent on $n$, so we write it by $\tilde{G}_{n}(m)$, that is,

$$
\tilde{G}_{n}(m)=\int_{m / \alpha}^{\theta} \frac{1}{\sqrt{-F(s)}} d s+(2 n+1) \int_{0}^{\theta} \frac{1}{\sqrt{-F(s)}} d s, \quad m \in(0, \alpha \theta) .
$$

It is easy to see that $\left\{\tilde{G}_{n}(m)\right\}_{n=0}^{\infty}$ is a strictly increasing sequence of $n$ for every $m \in(0, \alpha \theta)$, that is,

$$
\tilde{G}_{n}(m)<\tilde{G}_{n+1}(m), \quad m \in(0, \alpha \theta)
$$

(see Figure 3.2) and we can easily see that

$$
m_{2 n+1,1}^{*}<m_{2(n+1)+1,1}^{*}, \quad n=0,1,2, \ldots
$$

(see Figure 3.2). On the other hand, from (3.3) and (3.4) we have

$$
\begin{gathered}
\sqrt{\lambda_{2 n+1,2}}-\sqrt{\lambda_{2 n+1,1}}=\frac{1}{\sqrt{2}} \int_{0}^{\theta} \frac{1}{\sqrt{-F(s)}} d s-\frac{1}{\sqrt{2}} \int_{m / \alpha}^{\theta} \frac{1}{\sqrt{-F(s)}} d s, \quad m=m_{2 n+1,1}^{*}, \\
\sqrt{\lambda_{2(n+1)+1,2}}-\sqrt{\lambda_{2(n+1)+1,1}}=\frac{1}{\sqrt{2}} \int_{0}^{\theta} \frac{1}{\sqrt{-F(s)}} d s-\frac{1}{\sqrt{2}} \int_{m / \alpha}^{\theta} \frac{1}{\sqrt{-F(s)}} d s, \quad m=m_{2(n+1)+1,1}^{*} .
\end{gathered}
$$

Thus, combining (3.9) and (3.10) we obtain

$$
\sqrt{\lambda_{2 n+1,2}}-\sqrt{\lambda_{2 n+1,1}}<\sqrt{\lambda_{2(n+1)+1,2}}-\sqrt{\lambda_{2(n+1)+1,1}} .
$$


Also combining (3.3) and (3.4) we have

$$
\begin{aligned}
& \sqrt{\lambda_{2 n+1,2}}+\sqrt{\lambda_{2 n+1,1}} \\
& =\frac{4 n+3}{\sqrt{2}} \int_{0}^{\theta} \frac{1}{\sqrt{-F(s)}} d s+\frac{1}{\sqrt{2}} \int_{m / \alpha}^{\theta} \frac{1}{\sqrt{-F(s)}} d s, \quad m=m_{2 n+1,1}^{*}, \\
& \sqrt{\lambda_{2(n+1)+1,2}}+\sqrt{\lambda_{2(n+1)+1,1}} \\
& =\frac{4 n+7}{\sqrt{2}} \int_{0}^{\theta} \frac{1}{\sqrt{-F(s)}} d s+\frac{1}{\sqrt{2}} \int_{m / \alpha}^{\theta} \frac{1}{\sqrt{-F(s)}} d s, \quad m=m_{2(n+1)+1,1}^{*} .
\end{aligned}
$$

Since $0<m / \alpha<\theta$ for $m=m_{2 n+1,1}^{*}$, so we have

$$
\int_{m / \alpha}^{\theta} \frac{1}{\sqrt{-F(s)}} d s<\int_{0}^{\theta} \frac{1}{\sqrt{-F(s)}} d s, \quad m=m_{2 n+1,1}^{*},
$$

and then

$$
\begin{aligned}
\frac{1}{\sqrt{2}} \int_{m / \alpha}^{\theta} \frac{1}{\sqrt{-F(s)}} d s+\frac{4 n+3}{\sqrt{2}} \int_{0}^{\theta} \frac{1}{\sqrt{-F(s)}} d s \\
\quad<\frac{1}{\sqrt{2}} \int_{0}^{\theta} \frac{1}{\sqrt{-F(s)}} d s+\frac{4 n+3}{\sqrt{2}} \int_{0}^{\theta} \frac{1}{\sqrt{-F(s)}} d s .
\end{aligned}
$$

Now from (3.12) and (3.15) we obtain

$$
\sqrt{\lambda_{2 n+1,2}}+\sqrt{\lambda_{2 n+1,1}}<\frac{4 n+4}{\sqrt{2}} \int_{0}^{\theta} \frac{1}{\sqrt{-F(s)}} d s .
$$

On the other hand, by the positivity of

$$
\frac{3}{\sqrt{2}} \int_{0}^{\theta} \frac{1}{\sqrt{-F(s)}} d s+\frac{1}{\sqrt{2}} \int_{m / \alpha}^{\theta} \frac{1}{\sqrt{-F(s)}} d s, \quad m=m_{2(n+1)+1,1}^{*},
$$

and also from (3.13) and (3.16) we obtain

$$
\sqrt{\lambda_{2 n+1,2}}+\sqrt{\lambda_{2 n+1,1}}<\sqrt{\lambda_{2(n+1)+1,2}}+\sqrt{\lambda_{2(n+1)+1,1}} .
$$

Now combining (3.11) and (3.18) we have

$$
\lambda_{2 n+1,2}-\lambda_{2 n+1,1}<\lambda_{2(n+1)+1,2}-\lambda_{2(n+1)+1,1},
$$

thus, the proof is complete. 
4. Comparing the two cases $\rho=0$ and $\rho=\theta$. Now we compare $\lambda$ 's in the two cases $\rho=0$ and $\rho=\theta$ for any $n=0,1,2, \ldots$, and we are ready to prove the main theorem of this section.

THEOREM 4.1. Let $n=0,1,2, \ldots$, then

$$
\lambda_{2 n, 2}-\lambda_{2 n, 1}<\lambda_{2 n+1,2}-\lambda_{2 n+1,1}
$$

that is, the distance between $\lambda_{2 n, 1}\left(\theta, m_{2 n, 1}^{*}\right)$ and $\lambda_{2 n, 2}(\theta, 0)$ is less than the distance between $\lambda_{2 n+1,1}\left(0, m_{2 n+1,1}^{*}\right)$ and $\lambda_{2 n+1,2}(0,0)$.

Proof. Since $0<m / \alpha<\theta$ for $m=m_{2 n, 1}^{*}$, we have

$$
\int_{m / \alpha}^{\theta} \frac{1}{\sqrt{-F(s)}} d s<\int_{0}^{\theta} \frac{1}{\sqrt{-F(s)}} d s, \quad m=m_{2 n, 1}^{*},
$$

and then

$$
\frac{1}{\sqrt{2}} \int_{m / \alpha}^{\theta} \frac{1}{\sqrt{-F(s)}} d s+\frac{2 n}{\sqrt{2}} \int_{0}^{\theta} \frac{1}{\sqrt{-F(s)}} d s<\frac{2 n+1}{\sqrt{2}} \int_{0}^{\theta} \frac{1}{\sqrt{-F(s)}} d s, \quad m=m_{2 n, 1}^{*} .
$$

Now, from (4.3) and (2.3) we obtain

$$
\sqrt{\lambda_{2 n, 1}}<\frac{2 n+1}{\sqrt{2}} \int_{0}^{\theta} \frac{1}{\sqrt{-F(s)}} d s
$$

On the other hand, since $0<m / \alpha<\theta$ for $m=m_{2 n+1,1}^{*}$, then we have

$$
0<\frac{1}{\sqrt{2}} \int_{m / \alpha}^{\theta} \frac{1}{\sqrt{-F(s)}} d s, \quad m=m_{2 n+1,1}^{*},
$$

and then from (3.3) we have

$$
\sqrt{\lambda_{2 n, 1}}<\sqrt{\lambda_{2 n+1,1}}
$$

Also, we know that

$$
\begin{aligned}
\sqrt{2 \lambda_{2 n, 1}}=H(m)=\frac{m}{\sqrt{-F(m / \alpha)}}, & m=m_{2 n, 1}^{*}, \\
\sqrt{2 \lambda_{2 n+1,1}}=H(m)=\frac{m}{\sqrt{-F(m / \alpha)}}, & m=m_{2 n+1,1}^{*} .
\end{aligned}
$$

Now since function $H$ is one to one on interval $(0, \alpha \theta)$ (see Figure 4.1 ), we have

$$
m_{2 n, 1}^{*}<m_{2 n+1,1}^{*},
$$




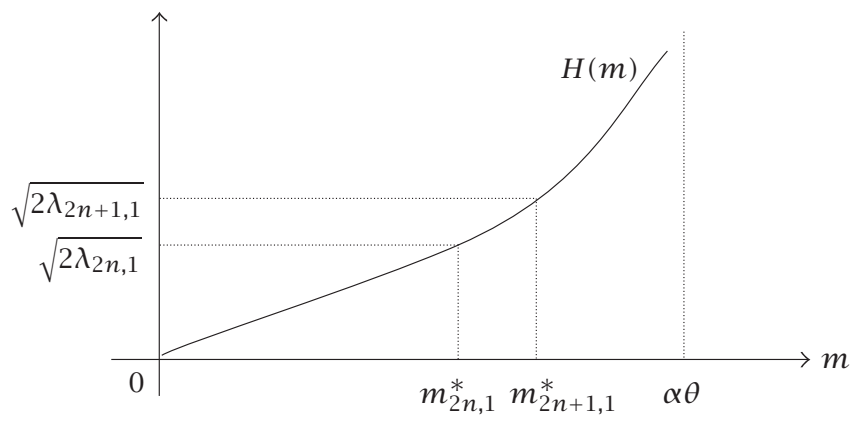

FigURE 4.1

so we obtain

$$
\int_{m_{2 n+1,1}^{*} / \alpha}^{\theta} \frac{1}{\sqrt{-F(s)}} d s<\int_{m_{2 n, 1}^{*} / \alpha}^{\theta} \frac{1}{\sqrt{-F(s)}} d s .
$$

Thus combining (2.10), (3.10), and (4.9) we obtain

$$
\sqrt{\lambda_{2 n, 2}}-\sqrt{\lambda_{2 n, 1}}<\sqrt{\lambda_{2 n+1,2}}-\sqrt{\lambda_{2 n+1,1}} .
$$

On the other hand, since $0<m / \alpha<\theta$ for $m=m_{2 n, 1}^{*}$, we have

$$
\int_{m / \alpha}^{\theta} \frac{1}{\sqrt{-F(s)}} d s<\int_{0}^{\theta} \frac{1}{\sqrt{-F(s)}} d s, \quad m=m_{2 n, 1}^{*},
$$

and then

$$
\frac{4 n+1}{\sqrt{2}} \int_{0}^{\theta} \frac{1}{\sqrt{-F(s)}} d s+\frac{1}{\sqrt{2}} \int_{m / \alpha}^{\theta} \frac{1}{\sqrt{-F(s)}} d s<\frac{4 n+2}{\sqrt{2}} \int_{0}^{\theta} \frac{1}{\sqrt{-F(s)}} d s, \quad m=m_{2 n, 1}^{*},
$$

and thus from (2.12) we obtain

$$
\sqrt{\lambda_{2 n, 2}}-\sqrt{\lambda_{2 n, 1}}<\frac{4 n+2}{\sqrt{2}} \int_{0}^{\theta} \frac{1}{\sqrt{-F(s)}} d s,
$$

and also from (4.13) we have

$$
\sqrt{\lambda_{2 n, 2}}+\sqrt{\lambda_{2 n, 1}}<\frac{4 n+3}{\sqrt{2}} \int_{0}^{\theta} \frac{1}{\sqrt{-F(s)}} d s+\frac{1}{\sqrt{2}} \int_{m / \alpha}^{\theta} \frac{1}{\sqrt{-F(s)}} d s, \quad m=m_{2 n+1,1}^{*} .
$$

So from (4.13) and (3.12), we obtain

$$
\sqrt{\lambda_{2 n, 2}}+\sqrt{\lambda_{2 n, 1}}<\sqrt{\lambda_{2 n+1,2}}+\sqrt{\lambda_{2 n+1,1}}
$$


and also from (4.15) and (4.10), we have

$$
\lambda_{2 n, 2}-\lambda_{2 n, 1}<\lambda_{2 n+1,2}-\lambda_{2 n+1,1}
$$

thus, the proof is complete.

\section{REFERENCES}

[1] V. Anuradha, C. Maya, and R. Shivaji, Positive solutions for a class of nonlinear boundary value problems with Neumann-Robin boundary conditions, J. Math. Anal. Appl. 236 (1999), no. 1, 94-124.

[2] V. Anuradha and R. Shivaji, Sign changing solutions for a class of superlinear multiparameter semi-positone problems, Nonlinear Anal. 24 (1995), no. 11, 1581-1596.

[3] A. Castro and R. Shivaji, Nonnegative solutions for a class of nonpositone problems, Proc. Roy. Soc. Edinburgh Sect. A 108 (1988), no. 3-4, 291-302.

[4] A. R. Miciano and R. Shivaji, Multiple positive solutions for a class of semipositone Neumann two-point boundary value problems, J. Math. Anal. Appl. 178 (1993), no. 1, 102-115.

G. A. Afrouzi: Department of Mathematics, Faculty of Basic Sciences, Mazandaran UNIVERSITY, BABOLSAR, IRAN

E-mail address: afrouzi@umz.ac.ir

M. Khaleghy Moghaddam: Department of Mathematics, Faculty of Basic SCiences, MAZANDARAN UNIVERSITY, BABOLSAR, IRAN 


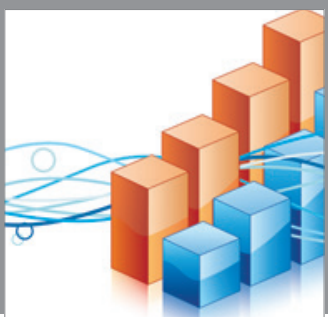

Advances in

Operations Research

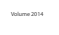

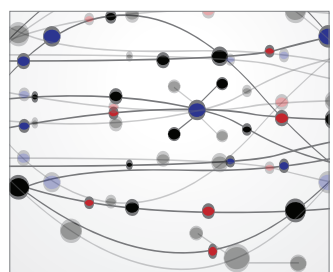

\section{The Scientific} World Journal
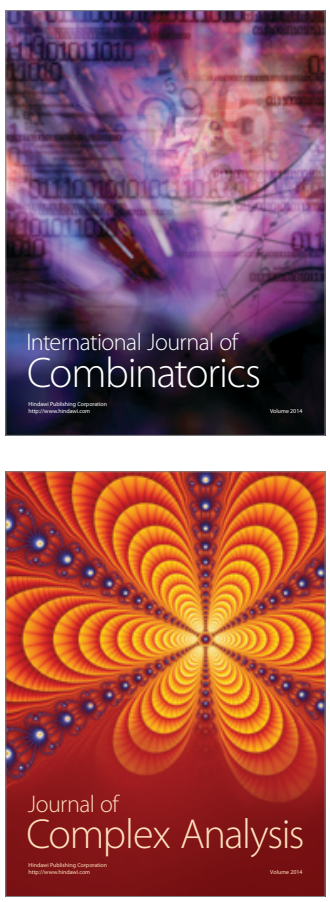

International Journal of

Mathematics and

Mathematical

Sciences
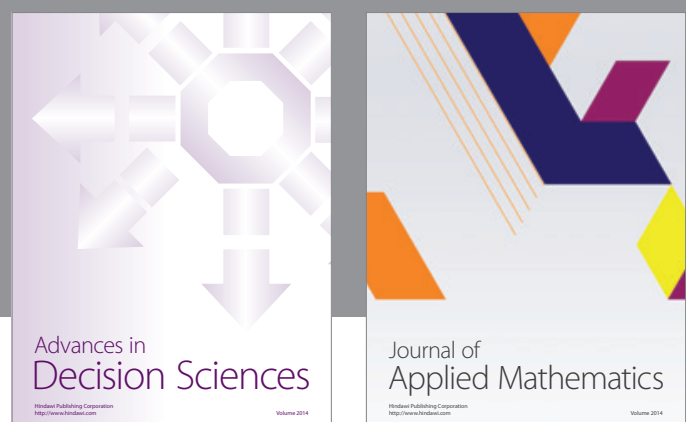

Journal of

Applied Mathematics
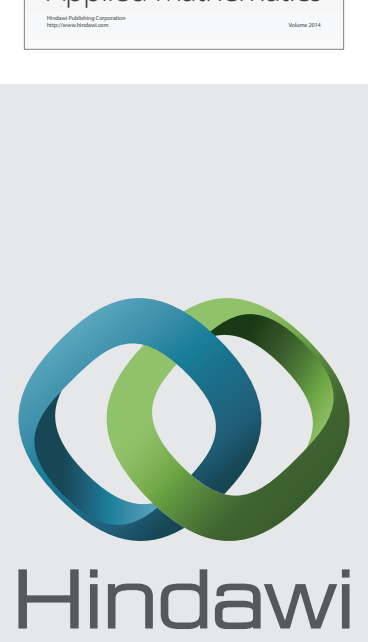

Submit your manuscripts at http://www.hindawi.com
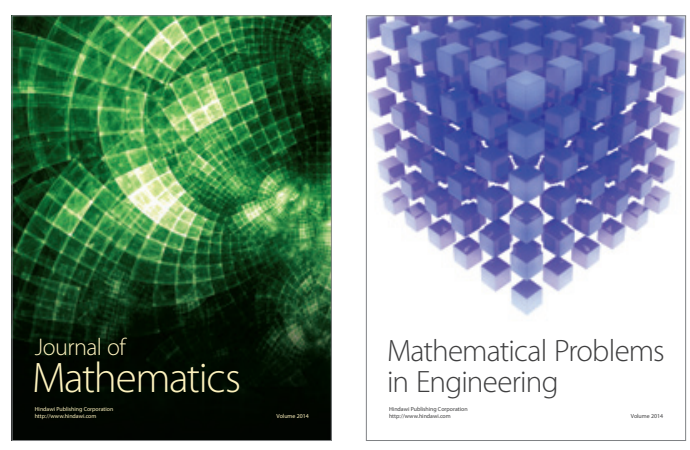

Mathematical Problems in Engineering
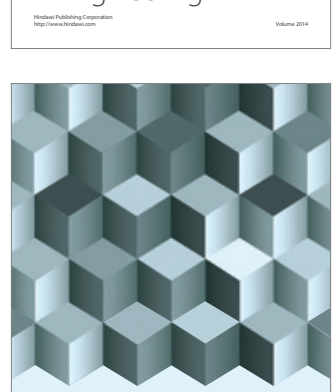

Journal of

Function Spaces
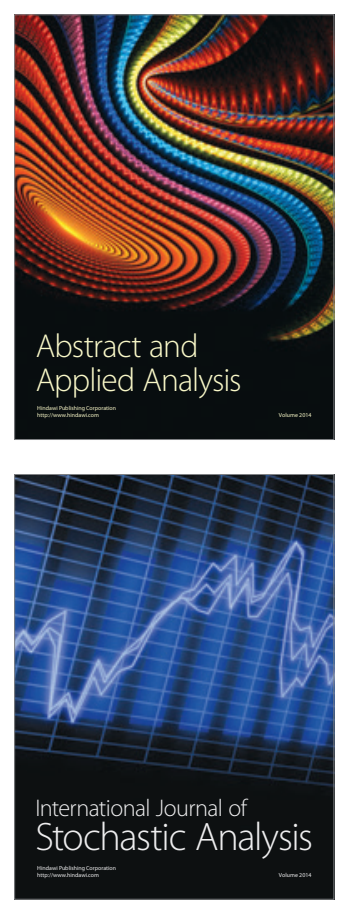

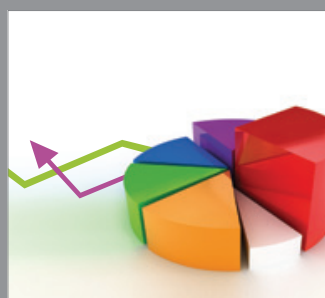

ournal of

Probability and Statistics

Promensencen
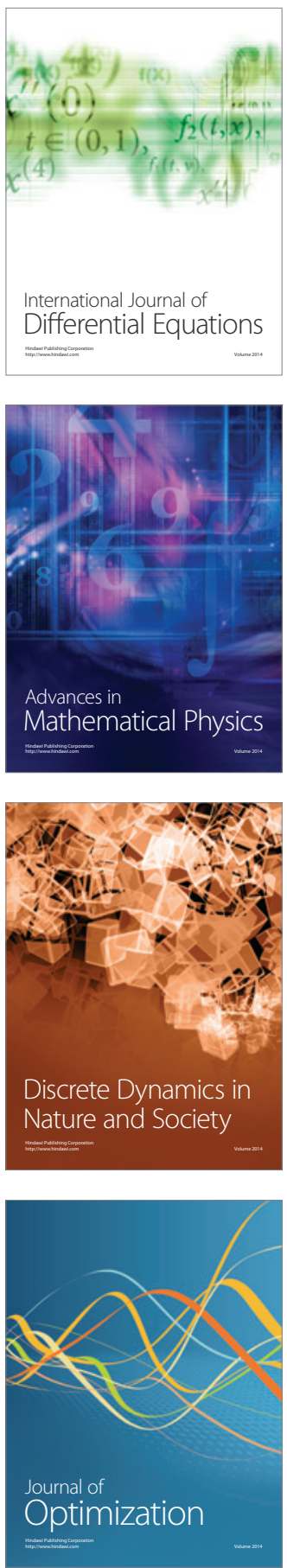